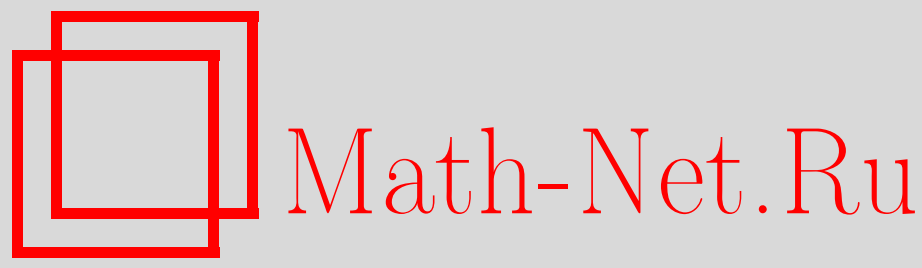

В. Г. Марихин, О некоторых решениях двумерных уравнений типа Шредингера в магнитном поле, ТМФ, 2011, том 168, номер 2, 219-226

DOI: https://doi.org/10.4213/tmf6676

Использование Общероссийского математического портала Math-Net.Ru подразумевает, что вы прочитали и согласны с пользовательским соглашением http://www.mathnet.ru/rus/agreement

Параметры загрузки:

IP: 54.157 .27 .8

26 апреля 2023 г., 12:35:10

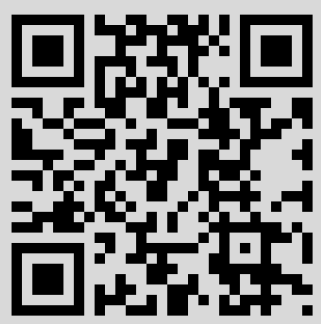


Том 168, № 2

август, 2011

(C) 2011 г.

\title{
В. Г. Марихин* \\ О НЕКОТОРЫХ РЕШЕНИЯХ ДВУМЕРНЫХ УРАВНЕНИЙ ТИПА ШРЕДИНГЕРА В МАГНИТНОМ ПОЛЕ
}

\begin{abstract}
Для построения новых решений двумерных уравнений типа Шредингера в магнитном поле используется метод одевания линейным дифференциальным оператором общего вида. В случае неединичной метрики проинтегрирован класс решений с разделяющимися переменными до одевания. Показано, в частности, что отношение коэффициентов перед операторами дифференцирования в случае единичной метрики удовлетворяет уравнению Хопфа. Установлена связь между решениями двумерного уравнения эйконала с единичной правой частью и решениями уравнения Хопфа.
\end{abstract}

Ключевые слова: метод одевания, квантовые операторы, уравнение Хопфа, уравнение эйконала.

\section{1. ВВЕДЕНИЕ}

Проблема построения новых решений двумерного уравнения Шредингера важна с точки зрения приложений и непосредственно связана с теорией интегрируемых систем. Такая задача, использующая преобразование Лапласа (один из вариантов метода одевания), рассматривалась в работе [1] (см. также [2]).

Метод одевания, использующий преобразования Дарбу [3], эффективен для построения решений интегрируемых, в том числе нелинейных, уравнений. В двумерном случае эти преобразования рассматривались, например, в работе [4] в применении к гиперболической системе. О другом варианте метода одевания - преобразовании Мутара - см. статью [5].

В дальнейшем мы следуем методике, предложенной в работе [6]. Обычно процедура одевания выглядит следующим образом. Пусть $H$ - некоторый оператор, а $\psi$ - его собственная функция: $H \psi=\lambda \psi$. Применяя одевающий оператор $S$ к функции $\psi$, получаем собственную функцию $\widehat{\psi}=S \psi$ одетого оператора $\widehat{H}, \widehat{H} \widehat{\psi}=\lambda \widehat{\psi}$, который удовлетворяет следующему уравнению:

$$
\widehat{H} S=S H .
$$

${ }^{*}$ Институт теоретической физики им. Л. Д. Ландау РАН, Москва, Россия. E-mail: mvg@itp.ac.ru 
В качестве начального мы берем гамильтониан $H$, в котором переменные разделены:

$$
H=t(x, y)\left(\partial_{x}^{2}+\partial_{y}^{2}+u_{1}(x)+u_{2}(y)\right)
$$

Мы не выписываем линейные по производным члены, поскольку они должны также зависеть от одной соответствующей переменной, и их можно устранить с помощью калибровочного преобразования. Оператор $S$ мы выбираем в виде

$$
S=\frac{1}{T(x, y)}\left(\partial_{x}+\phi(x, y) \partial_{y}+f(x, y)\right) .
$$

Здесь $T(x, y), \phi(x, y), f(x, y)$ - произвольные функции, причем $T(x, y)$ является чисто калибровочной функцией и выбирается из соображения удобства. Оператор $S$ представляет собой наиболее общий двумерный линейный оператор, и в этом состоит основное отличие настоящей работы от цитируемых выше работ [1], [4], где оператор одевания соответствует случаю $\phi= \pm i$. Чтобы сохранить вещественность преобразований, мы вынуждены выбрать одеваемый и одетый гамильтониан в виде, в котором оператор импульса заменяется на обычную производную, $-i \nabla \rightarrow \nabla$, поэтому мы говорим не об уравнении Шредингера, а об одевании оператора типа Шредингера. Чтобы исследовать непосредственно уравнение Шредингера с помощью нашего подхода, необходимо чисто мнимые нетривиальные решения уравнения Хопфа. На данный момент нам неизвестны такие решения.

Мы действуем уравнением (1) на вспомогательную функцию и требуем чтобы все члены, содержащие производные, обращались в ноль. Обращение в ноль коэффициентов при $\psi_{x x x}, \psi_{x x y}, \psi_{x y y}, \psi_{y y y}$ приводит к следующему виду одетого гамильтониана:

$$
\widehat{H}=t(x, y)\left(\left(\partial_{x}-A_{1}(x, y)\right)^{2}+\left(\partial_{y}-A_{2}(x, y)\right)^{2}+U(x, y)\right) .
$$

Коэффициенты при $\psi_{x x}, \psi_{y y}$ определяют векторные потенциалы $A_{1}, A_{2}$ соответственно, а коэффициент при $\psi_{x y}$ приводит к интересному соотношению

$$
t_{x}+\phi t_{y}-2 \frac{\phi_{y}-\phi \phi_{x}}{\phi^{2}+1} t=0 .
$$

Подстановка

$$
t=\frac{w(Z)}{Z_{x}^{2}+Z_{y}^{2}}, \quad \phi=-\frac{Z_{x}}{Z_{y}},
$$

где $Z$ - произвольная функция от $x$ и $y$, а $w$ - произвольная функция от $Z$, полностью интегрирует соотношение (5). Обращение в ноль коэффициента, например, при $\psi_{y}$ определяет одетый потенциал $U$; дальнейшее вычисление коэффициента при $\psi_{x}$ приводит к уравнению для функции $f$, которое можно записать в виде $\tilde{f}=f Z_{y}$, при этом

$$
\begin{aligned}
Z_{x} \tilde{f}_{x} & +Z_{y} \tilde{f}_{y}+\frac{Z_{x}^{2}-Z_{y}^{2}}{Z_{x}^{2}+Z_{y}^{2}}\left(Z_{y y}-Z_{x x}-4 Z_{x} Z_{y} Z_{x y}\right) \tilde{f}+ \\
& +\frac{1}{2}\left(-Z_{y} Z_{x x x}+Z_{x} Z_{x x y}-Z_{y} Z_{x y y}+Z_{x} Z_{y y y}\right)+ \\
& +\left(Z_{x x}-Z_{y y}\right) Z_{x} Z_{y}+Z_{x y}\left(Z_{y}^{2}-Z_{x}^{2}\right)=0
\end{aligned}
$$


Мы ищем решение этого уравнения в виде

$$
\tilde{f}=\frac{1}{2}\left(\frac{Z_{y}}{Z_{x}} Z_{x x}-\frac{Z_{x}}{Z_{y}} Z_{y y}\right)+\tilde{f}_{0},
$$

тогда уравнение для $f_{0}$ не имеет локальных по $Z$ (т. е. зависящих только от $Z$ и его производных) решений, поэтому мы полагаем $f_{0}=0$, получая следующее уравнение: $\partial_{x} \partial_{y} \ln \phi=0$. При этом

$$
Z=L\left(\int d x F(x)-\int d y G(y)\right) .
$$

Уравнение, получающееся при обращении в ноль коэффициента при $\psi$, имеет вид

$$
\begin{aligned}
u(x, y) & -\frac{1}{2} \frac{F^{\prime \prime}(x)}{F(x)}+\frac{3}{4} \frac{\left(F^{\prime}(x)\right)^{2}}{F^{2}(x)}-\frac{1}{2} \frac{G^{\prime \prime}(y)}{G(y)}+\frac{3}{4} \frac{\left(G^{\prime}(y)\right)^{2}}{G^{2}(y)}+ \\
& +\left(F^{2}(x)+G^{2}(y)\right) R^{\prime}\left(\int d x F(x)-\int d y G(y)\right)=0 .
\end{aligned}
$$

При $R^{\prime}=\mu^{2}=$ const переменные разделены, $u(x, y)=u_{1}(x)+u_{2}(y)$, $u_{1}(x)=\frac{1}{2} \frac{F^{\prime \prime}(x)}{F(x)}-\frac{3}{4} \frac{\left(F^{\prime}(x)\right)^{2}}{F^{2}(x)}-\mu^{2} F(x)^{2}, \quad u_{2}(y)=\frac{1}{2} \frac{G^{\prime \prime}(y)}{G(y)}-\frac{3}{4} \frac{\left(G^{\prime}(y)\right)^{2}}{G^{2}(y)}-\mu^{2} G^{2}(y)$.

Волновая функция $\psi(x, y)=\psi_{1}(x) \psi_{2}(y)$, отвечающая нулевому собственному значению, задается формулами

$$
\begin{aligned}
& \psi_{1}(x)=\frac{a_{1} \exp \left\{\mu \int d x F(x)\right\}+a_{2} \exp \left\{-\mu \int d x F(x)\right\}}{\sqrt{F(x)}}, \\
& \psi_{2}(y)=\frac{b_{1} \exp \left\{\mu \int d y G(y)\right\}+b_{2} \exp \left\{-\mu \int d y G(y)\right\}}{\sqrt{G(y)}} .
\end{aligned}
$$

Одетая волновая функция $\widehat{\psi}$ записывается как

$$
\begin{aligned}
\widehat{\psi}=S \psi= & \frac{2 \mu}{\sqrt{F(x)} \sqrt{G(y)}}\left(a_{1} b_{1} \exp \left\{\mu\left(\int d x F(x)+\int d y G(y)\right)\right\}-\right. \\
& \left.-a_{2} b_{2} \exp \left\{-\mu\left(\int d x F(x)+\int d y G(y)\right)\right\}\right) .
\end{aligned}
$$

Магнитное поле есть

$$
\begin{aligned}
B= & \frac{F(x) G(y)}{\left(F^{2}(x)+G^{2}(y)\right)^{2}}\left(\left(G^{\prime}(y)\right)^{2}-\left(F^{\prime}(x)\right)^{2}\right)+ \\
& +\frac{1}{2} \frac{1}{F^{2}(x)+G^{2}(y)}\left(G(y) F^{\prime \prime}(x)-F(x) G^{\prime \prime}(y)\right) .
\end{aligned}
$$

Потенциал равен

$$
\begin{aligned}
U(x, y)= & u(x, y)-\frac{G^{2}(y)}{F(x)} \frac{F^{\prime \prime}(x)}{F^{2}(x)+G^{2}(y)}-\frac{F^{2}(x)}{G(y)} \frac{G^{\prime \prime}(y)}{F^{2}(x)+G^{2}(y)}+ \\
& +\frac{G^{2}(y)}{F^{2}(x)} \frac{G^{2}(y)+3 F^{2}(x)}{\left(F^{2}(x)+G^{2}(y)\right)^{2}}\left(F^{\prime}(x)\right)^{2}+\frac{F^{2}(x)}{G^{2}(y)} \frac{F^{2}(x)+3 G^{2}(y)}{\left(F^{2}(x)+G^{2}(y)\right)^{2}}\left(G^{\prime}(y)\right)^{2} .
\end{aligned}
$$


Таким образом, функции $F(x), G(y)$ полностью определяют потенциал и магнитное поле после одевания и потенциал до одевания (магнитное поле до одевания равно нулю). Было бы интересно получить после одевания гамильтониан с заданными свойствами, например такой, что $U=0, B \neq 0$. Решение этой задачи в общем случае неизвестно, однако есть один нетривиальный пример, задающий функцию $G$ неявно:

$$
F(x)=1, \quad \int^{G(y)} d a \frac{1-a^{2}}{1+a^{2}} \frac{1}{\sqrt{\left(a^{2}-1\right) a\left(4 \mu^{2} a+c_{1}\right)}}=y+c_{2} .
$$

\section{2. СЛУЧАЙ $t=1$. ОДЕВАНИЕ ОПЕРАТОРА ТИПА ШРЕДИНГЕРА}

В случае $t=1$ может быть проведено одевание оператора типа Шредингера (с единичной метрикой) с ненулевым начальным магнитным полем, при этом гамильтонианы до и после одевания имеют вид

$$
\begin{aligned}
H_{0} & =\left(\partial_{x}-a_{1}(x, y)\right)^{2}+\left(\partial_{y}-a_{2}(x, y)\right)^{2}+u(x, y), \\
H & =\left(\partial_{x}-A_{1}(x, y)\right)^{2}+\left(\partial_{y}-A_{2}(x, y)\right)^{2}+U(x, y),
\end{aligned}
$$

причем одевающий оператор $S$ должен иметь калибровочно-инвариантную форму:

$$
S=\left(\partial_{x}-a_{1}(x, y)\right)+\phi(x, y)\left(\partial_{y}-a_{2}(x, y)\right)+f(x, y) .
$$

Отметим важное обстоятельство: из уравнения (5) при $t=1$ получаем, что функция $Z$ удовлетворяет двумерному уравнению эйконала

$$
Z_{x}^{2}+Z_{y}^{2}=w(Z)
$$

а функция $\phi=-Z_{x} / Z_{y}$ удовлетворяет уравнению Хопфа $\phi_{y}=\phi \phi_{x}$. Конечно, по заданному решению уравнения Хопфа также можно построить решение двумерного уравнения эйконала (8) следующим образом.

Выбираем общее решение уравнения Хопфа в виде $z(\phi)=x+y \phi$, следовательно,

$$
\phi_{x x}=-z^{\prime \prime}(\phi) \phi_{x}^{3}, \quad \phi_{x x x}=-z^{\prime \prime \prime}(\phi) \phi_{x}^{4}+3\left(z^{\prime \prime}(\phi)\right)^{2} \phi_{x}^{5} .
$$

Для дальнейших вычислений удобно сделать следующую замену переменных: $(x, y) \rightarrow\left(\phi(x, y), \phi_{x}(x, y)\right)=(\phi, \eta):$

$$
\begin{aligned}
x & =z(\phi)-\phi\left(z^{\prime}(\phi)-\frac{1}{\eta}\right), & y & =z^{\prime}(\phi)-\frac{1}{\eta}, \\
\partial_{x} & =\eta \partial_{\phi}-\eta^{3} z^{\prime \prime}(\phi) \partial_{\eta}, & \partial_{y} & =\eta \phi \partial_{\phi}+\eta^{2}\left(1-\eta \phi z^{\prime \prime}(\phi)\right) \partial_{\eta} .
\end{aligned}
$$

Введем величину

$$
\xi=\frac{\sqrt{1+\phi^{2}}}{\eta}-\int d \phi z^{\prime \prime}(\phi) \sqrt{1+\phi^{2}}
$$

которая является решением уравнения эйконала с единичной правой частью, т. е. $\left(\partial_{x} \xi\right)^{2}+\left(\partial_{y} \xi\right)^{2}=1$. Оказывается, что общее решение уравнения $\partial_{x} F+\phi \partial_{y} F=0$ есть функция $F(\xi)$. Выберем функцию $F$ такую, что $\left(F^{\prime}(Z)\right)^{2}=w(Z)$, тогда эта функция и функция $z(\phi)$ задают решение уравнения эйконала $(8)$. 
Вычислим магнитное поле в одетом гамильтониане $H$ в зависимости от поля в гамильтониане $H_{0}$ :

$$
B=\partial_{x} A_{2}-\partial_{y} A_{1}=B_{0}+\partial_{x} \partial_{y} \ln \phi=B_{0}+\partial_{x}^{2} \phi, \quad B_{0}=\partial_{x} a_{2}-\partial_{y} a_{1} .
$$

Потенциалы имеют вид

$$
\begin{aligned}
u & =\frac{1}{1+\phi^{2}}\left(-f^{2}+\left(\partial_{x}+\phi \partial_{y}-\eta \phi\right) f-\frac{1}{4} \eta^{2}+G(\xi)\right), \\
U & =\frac{1}{1+\phi^{2}}\left(-f^{2}-\left(\partial_{x}+\phi \partial_{y}-\eta \phi\right) f-\frac{1}{4} \eta^{2}+G(\xi)\right),
\end{aligned}
$$

где $G(\xi)$ - произвольная функция. Магнитные поля суть

$$
\begin{aligned}
B_{0} & =-\frac{1}{2} \partial_{x}^{2} \phi-\frac{1}{1+\phi^{2}}\left(\partial_{y}-\phi \partial_{x}-\phi_{x}\right) f \\
B & =\frac{1}{2} \partial_{x}^{2} \phi-\frac{1}{1+\phi^{2}}\left(\partial_{y}-\phi \partial_{x}-\phi_{x}\right) f
\end{aligned}
$$

Аналог одевающей цепочки [7] можно построить, используя уравнения (10), (11):

$$
u\left(\hat{f}, \hat{z}, \widehat{\partial_{x}}, \widehat{\partial_{y}}, \widehat{G(\xi)}\right)=U\left(f, z, \partial_{x}, \partial_{y}, G(\xi)\right), \quad B_{0}\left(\hat{f}, \hat{z}, \widehat{\partial_{x}}, \widehat{\partial_{y}}\right)=B\left(f, z, \partial_{x}, \partial_{y}\right)
$$

Необходимо подчеркнуть следующее: в этом разделе мы считаем, что все функции зависят от $\phi, \eta$ (эти переменные не преобразуются при одевании), поэтому операторы $\partial_{x}, \partial_{y}$ определяются формулой $(9)$, при одевании эти операторы преобразуются следующим образом:

$$
z(\phi) \rightarrow \hat{z}(\phi) \Rightarrow \partial_{x} \rightarrow \widehat{\partial_{x}}, \quad \partial_{y} \rightarrow \widehat{\partial_{y}}
$$

Приведем пример с постоянным одетым потенциалом:

$$
z(\phi)=\mu \sqrt{1+\phi^{2}}, \quad s(\phi, \eta)=2 \mu \frac{\eta^{2}}{\sqrt{1+\phi^{2}}}-\frac{1}{16 \mu} \sqrt{1+\phi^{2}}
$$

при этом

$$
\begin{aligned}
B_{0} & =-\frac{\eta}{16 \mu} \frac{1}{\sqrt{1+\phi^{2}}}-\frac{3 \mu}{2} \frac{\eta^{3}}{\sqrt{\left(1+\phi^{2}\right)^{3}}}, & B & =-\frac{\eta}{16 \mu} \frac{1}{\sqrt{1+\phi^{2}}}-\frac{\mu}{2} \frac{\eta^{3}}{\sqrt{\left(1+\phi^{2}\right)^{3}}}, \\
u & =-\frac{1}{256 \mu^{2}}+8 \mu^{2} \frac{\eta^{2}}{\sqrt{\left(1+\phi^{2}\right)^{3}}}, & U & =-\frac{1}{256 \mu^{2}} .
\end{aligned}
$$

В случае $\phi=$ const $\neq \pm i$ получаем

$$
u(x, y)=\frac{1}{\phi^{2}+1}\left(D_{+} f-f^{2}\right), \quad U(x, y)=-\frac{1}{\phi^{2}+1}\left(D_{+} f+f^{2}\right), \quad D_{+}=\partial_{x}+\phi \partial_{y},
$$

т. е. одевающую цепочку (ср. с [7]), причем

$$
\begin{gathered}
A_{1}(x, y)=a_{1}(x, y), \quad A_{2}(x, y)=a_{2}(x, y), \\
b(x, y)=\partial_{x} a_{2}-\partial_{y} a_{1}=\frac{D_{-} f}{\phi^{2}+1}, \quad D_{-}=\partial_{x}-\phi \partial_{y} .
\end{gathered}
$$




\section{3. ОБСУЖДЕНИЕ}

В настоящей работе рассмотрено одевание операторов типа Шредингера в магнитном поле. В данном варианте метода одевания магнитное поле изменяется в процессе одевания. Рассмотрены операторы типа Шредингера как с неединичной метрикой $(t \neq 1)$, так и с единичной метрикой $(t=1)$. В первом случае при некоторых естественных ограничениях и при нулевом начальном магнитном поле класс решений по существу сводится к системам с разделенными переменными. Все ответы в этом случае выписываются в простой и явной форме, хотя одетый гамильтониан содержит потенциал и магнитное поле с неразделяющимися переменными. Важно установить, можно ли снять наложенные ограничения или полученный ответ является достаточно общим. Во втором случае все вычисления выполнены с ненулевыми начальными и конечными магнитными полями и потенциалами.

Было бы интересно провести классификацию возможных решений в этих двух случаях, например представляет определенный интерес случай, когда потенциал одетого гамильтониана равен нулю, а магнитное поле отлично от нуля.

Соотношение (5) интересно тем, что при $t=1$ позволяет получить решение двумерного уравнения эйконала вида (8) по заданному решению уравнения Хопфа, и наоборот - решение уравнения эйконала вида (8) задает явное решение уравнения Хопфа. Представляет определенный интерес классификация получаемых таким образом решений.

\section{Случай анизотропных масс}

ПРИЛОЖЕНИЕ

Рассмотрим гамильтонианы с анизотропными массами:

$$
\begin{aligned}
H & =t(x, y)\left(a(x) \partial_{x}^{2}+b(y) \partial_{y}^{2}+u(x, y)\right) \\
\widehat{H} & =t(x, y)\left(a(x)\left(\partial_{x}-A_{1}(x, y)\right)^{2}+b(y)\left(\partial_{y}-A_{2}(x, y)\right)^{2}+U(x, y)\right) .
\end{aligned}
$$

Дальнейшие вычисления полностью аналогичны изотропному случаю $a=1, b=1$, рассмотреному ранее, поэтому приведем только окончательные формулы. Получаем

$$
\begin{aligned}
S & =\frac{1}{F(x)} \partial_{x}+\frac{1}{G(y)} \partial_{y}+\frac{1}{2} \frac{F^{\prime}(x)}{F^{2}(x)}+\frac{1}{2} \frac{G^{\prime}(y)}{G^{2}(y)}, \\
t & =\frac{1}{\left(L^{\prime}\right)^{2}\left(\int d x F(x)-\int d y G(y)\right)\left(a(x) F^{2}(x)+b(y) G^{2}(y)\right)} .
\end{aligned}
$$

Векторный потенциал задается формулами

$$
\begin{aligned}
A_{1}= & \frac{1}{a(x) F^{2}(x)+b(y) G^{2}(y)} \times \\
& \times\left[F(x) G^{\prime}(y) b(y)+\frac{1}{2} F(x) G(y) b^{\prime}(y)-\left(\frac{F^{\prime}(x)}{F(x)}+\frac{1}{2} \frac{a^{\prime}(x)}{a(x)}\right) G^{2}(y) b(y)\right], \\
A_{2}= & \frac{1}{a(x) F^{2}(x)+b(y) G^{2}(y)} \times \\
& \times\left[G(y) F^{\prime}(x) a(x)+\frac{1}{2} F(x) G(y) a^{\prime}(x)-\left(\frac{G^{\prime}(y)}{G(y)}+\frac{1}{2} \frac{b^{\prime}(y)}{b(y)}\right) F^{2}(x) a(x)\right] .
\end{aligned}
$$


Магнитное поле равно

$$
\begin{aligned}
B= & \frac{a(x) G(y) F^{\prime \prime}(x)-b(y) F(x) G^{\prime \prime}(y)}{a(x) F^{2}(x)+b(y) G^{2}(y)}-2 F(x) G(y) \frac{a^{2}(x)\left(F^{\prime}(x)\right)^{2}-b^{2}(y)\left(G^{\prime}(y)\right)^{2}}{\left(a(x) F^{2}(x)+b(y) G^{2}(y)\right)^{2}}- \\
& -\frac{1}{2} \frac{a^{\prime}(x) G(y) F^{\prime}(x)\left(a(x) F^{2}(x)-3 b(y) G^{2}(y)\right)^{2}}{\left(a(x) F^{2}(x)+b(y) G^{2}(y)\right)^{2}}- \\
& -\frac{1}{2} \frac{b^{\prime}(y) G^{\prime}(y) F(x)\left(3 a(x) F^{2}(x)-b(y) G^{2}(y)\right)^{2}}{\left(a(x) F^{2}(x)+b(y) G^{2}(y)\right)^{2}}+ \\
& +\frac{1}{2} \frac{F(x) G(y)\left(a^{\prime \prime}(x)-b^{\prime \prime}(y)\right)}{a(x) F^{2}(x)+b(y) G^{2}(y)}-\frac{1}{2} \frac{F(x) G(y)\left(\left(a^{\prime}(x)\right)^{2} F^{2}(x)-\left(b^{\prime}(y)\right)^{2} G^{2}(y)\right)}{\left(a(x) F^{2}(x)+b(y) G^{2}(y)\right)^{2}} .
\end{aligned}
$$

Потенциал до одевания записывается как

$$
\begin{aligned}
u(x, y)= & a(x) \frac{1}{2} \frac{F^{\prime \prime}(x)}{F(x)}+b(y) \frac{1}{2} \frac{G^{\prime \prime}(y)}{G(y)}-a(x) \frac{3}{4} \frac{\left(F^{\prime}(x)\right)^{2}}{F^{2}(x)}-b(y) \frac{3}{4} \frac{\left(G^{\prime}(y)\right)^{2}}{G^{2}(y)}+ \\
& +\left(a(x) F^{2}(x)+b(y) G^{2}(y)\right) R\left(\int d x F(x)-\int d y G(y)\right) .
\end{aligned}
$$

Потенциал после одевания есть

$$
\begin{aligned}
U(x, y)= & u(x, y)-\frac{a(x) b(y)}{F(x)} \frac{G^{2}(y) F^{\prime \prime}(x)}{a(x) F^{2}(x)+b(y) G^{2}(y)}- \\
& -\frac{a(x) b(y)}{G(y)} \frac{F^{2}(x) G^{\prime \prime}(y)}{a(x) F^{2}(x)+b(y) G^{2}(y)}+ \\
& +a(x) b(y)\left(F^{\prime}(x)\right)^{2} \frac{G^{2}(y)}{F^{2}(x)} \frac{3 a(x) F^{2}(x)+b(y) G^{2}(y)}{\left(a(x) F^{2}(x)+b(y) G^{2}(y)\right)^{2}}+ \\
& +a(x) b(y)\left(G^{\prime}(y)\right)^{2} \frac{F^{2}(x)}{G^{2}(y)} \frac{a(x) F^{2}(x)+3 b(y) G^{2}(y)}{\left(a(x) F^{2}(x)+b(y) G^{2}(y)\right)^{2}}+ \\
& +\frac{1}{2} a^{\prime}(x) b(y) F^{\prime}(x) \frac{G^{2}(y)}{F(x)} \frac{3 a(x) F^{2}(x)-b(y) G^{2}(y)}{\left(a(x) F^{2}(x)+b(y) G^{2}(y)\right)^{2}}- \\
& -\frac{1}{2} a(x) b^{\prime}(y) G^{\prime}(y) \frac{F^{2}(x)}{G(y)} \frac{a(x) F^{2}(x)-3 b(y) G^{2}(y)}{\left(a(x) F^{2}(x)+b(y) G^{2}(y)\right)^{2}}+ \\
& +\frac{1}{4}\left(a^{\prime}(x)\right)^{2} \frac{b(y) G^{2}(y)}{a(x)} \frac{3 a(x) F^{2}(x)+b(y) G^{2}(y)}{\left(a(x) F^{2}(x)+b(y) G^{2}(y)\right)^{2}}+ \\
& +\frac{1}{4}\left(b^{\prime}(y)\right)^{2} \frac{a(x) F^{2}(x)}{b(y)} \frac{a(x) F^{2}(x)+3 b(y) G^{2}(y)}{\left(a(x) F^{2}(x)+b(y) G^{2}(y)\right)^{2}}- \\
& -\frac{1}{2} \frac{a(x) b^{\prime \prime}(y) F^{2}(x)+a^{\prime \prime}(x) b(y) G^{2}(y)}{a(x) F^{2}(x)+b(y) G^{2}(y)} .
\end{aligned}
$$

2 Теоретическая и математическая физика, т. 168, № 2, 2011 г. 
Отметим, что пример, приведенный в работе [6] (см. также [8]), относится к данному классу одевания с разделяющимися переменными, при этом

$$
\begin{gathered}
a(x)=x^{2}-1, \quad b(y)=1-y^{2}, \quad F(x)=1, \quad G(y)=-1, \\
R(x)=\frac{1}{x}, \quad u(x, y)=y-x, \quad L(x)=2 \sqrt{x} .
\end{gathered}
$$

Благодарности. Автор благодарен А. Б. Шабату, В. Э. Адлеру и В. В. Соколову за внимание к работе и полезные обсуждения. Работа выполнена при частичной финансовой поддержке РФФИ (грант № 10-01-00088).

\section{Список литературы}

[1] S.P. Novikov, A.P. Veselov, "Exactly solvable 2-dimensional Schrödinger opeators and Laplace transformations", Solitons, Geometry and Topology: On the Crossroads, Amer. Math. Soc. Transl. Ser. 2, 179, eds. V. M. Buchstaber, S. P. Novikov, AMS, Providence, RI, 1997, 109-132, arXiv: math-ph/0003008.

[2] E. V. Ferapontov, A.P. Veselov, J. Math. Phys., 42:2 (2001), 590-607, arXiv: math-ph/0007034.

[3] G. Darboux, Lecons sur la Théorie Générale des Surfaces et les Applications Geométriques du Calcul Infinitésimal. Première Partie. Généralités. Coordonnées Curvilignes. Surfaces minima, Réimpression de la deuxième edition de 1914, Chelsea, New York, 1972.

[4] А. Б. Шабат, ТМФ, 103:1 (1995), 170-175.

[5] И. А. Тайманов, С. П. Царев, ТМФ, 157:2 (2008), 188-207, arXiv: 0801.3225.

[6] В. Г. Марихин, ТМФ, 161:3 (2009), 327-331.

[7] А. П. Веселов, А.Б. Шабат, Функи. анализ и его прил., 27:2 (1993), 1-21.

[8] М.А. Скворцов, П. М. Островский, Писъма в ЖЭЭТФ, 85:1 (2007), 79-83, arXiv: cond-mat/0604631. 